FERMILAB-FN-1099-AD

\title{
Injection considerations for PIP-III Linac to Main Injector
}

\author{
David Neuffer ${ }^{a}$
}

${ }^{a}$ Fermilab, PO Box 500, Batavia IL 60510 USA

\begin{abstract}
The PIP-III linac produces $8 \mathrm{GeV} \mathrm{H}^{-}$beam for injection into the Recycler or Main Injector (MI). Stripping to $\mathrm{H}+$ is needed, in a system that is vulnerable to magnetic stripping of $\mathrm{H}$ - in magnetic fields. The High power beam will heat stripping foils, perhaps unacceptably. The injection pulse is much longer than the MI circumference, so multiturn pulses with resulting heating and beam diffusion are required. We discuss design considerations for the injection scenario, the stripper foil and its location, foil heating and related beam loss issues.
\end{abstract}

Keywords: muon, beams, PIP-II

\section{INTRODUCTION}

The PIP-II project will provide a $800 \mathrm{MeV}$ proton beam with $\mathrm{cw}$ capability, with beam power up to the MW level available for user experiments.[1] However, the amount of beam that can be transmitted to the Main Injector (MI) is limited by the $0.8-8.0 \mathrm{GeV}$ Booster capacity. The next Fermilab upgrade should include a replacement for the Booster. The project-X design proposal included some options for that replacement, based on a continuation of the $800 \mathrm{MeV}$ linac to 2-3 GeV followed by either a Rapid Cycling Synchrotron (RCS) or continuing the Linac to 8 $\mathrm{GeV}$.[2] While an $8 \mathrm{GeV}$ Linac would be expected to be very expensive, it may be made relatively affordably by using relatively inexpensive ILC-style cryomodules that use $1300 \mathrm{MHz}$ SRF cavities, that have already been designed and mass-produced.

In this note we will focus on the $8 \mathrm{GeV}$ Linac option. We begin with some discussion of the beam requirements and potential layouts for the Linac. Constraints on accelerating gradients and magnetic fields are discussed. We then progress to discussion of injection from the $8 \mathrm{GeV}$ Linac into the Recycler ring or MI. The injection process involves foil stripping of the incoming $\mathrm{H}^{-}$beam to obtain multiturn injection. Potential problems exist in magnetic stripping of $\mathrm{H}^{-}$beam, stripping efficiency, foil heating, foil damage and radiation in the injection region. An optimum "beam painting' strategy is needed. First calculations of these effects are obtained and critical difficulties are discussed. As with other high-intensity injection examples (SNS, PIP-III RCS, JPARC), foil heating approaches the limits of present technologies. Extensions of existing technologies, such as rotating foils, or newer technologies such as laser-assisted stripping could extend those limts.

\section{LINAC SCENARIOS}

The initial design specification for the PIP-III upgrade is that it should enable at least $\sim 2.5$ MW from the MI. With a $120 \mathrm{GeV}$ beam energy and a MI period of $1.2 \mathrm{~s}$, this requires $1.5625^{*} 10^{15} \mathrm{p} / \mathrm{cycle}$, or $25 \mathrm{ma}-\mathrm{ms}$ of injected beam. The $800 \mathrm{MeV}$ beam PIP-II beam can provide up to $2 \mathrm{ma}$ of $\mathrm{cw}$ beam, so $12.5 \mathrm{~ms}$ of injection would be sufficient. More beam would of course be desirable.

Scenarios for an $8 \mathrm{GeV}$ Linac scenario were developed within the project X program.[3] Fig. 1 shows a possible scenario. The $800 \mathrm{MeV}$ Linac is extended to $\sim 1 \mathrm{GeV}$. The beam exiting that Linac is bent at a steep angle into a $1 \rightarrow 3 \mathrm{GeV}$ linac ( $280 \mathrm{~m}$ long). In the Project X scenario that linac is a cw linac that uses the same $650 \mathrm{MHz}$ cryomodules as the end of the PIP-II linac. The beam then goes through a bend of approximately $100^{\circ}$ to be pointed toward injection into the Recycler. A $\sim 390 \mathrm{~m} 3 \rightarrow 8 \mathrm{GeV}$ pulsed linac, consisting of ILC $1300 \mathrm{MHz}$ cryomodules takes the beam toward the MI. Parameters of the different linac components are shown in Table 1.

This manuscript has been authored by Fermi Research Alliance, LLC under Contract No. DE-AC02-07CH11359 with the U.S. Department of Energy, Office of Science, Office of High Energy Physics. 
The curves away from the MI and back toward the MI are needed to fit the somewhat longer linac segments into the relatively short space between PIP-II and the MI. The current PIP-II is moved $\sim 100$ feet to the right from the position shown in Fig. 1. This places it slightly further from the MI injection point which can be used to fit a slightly larger curved linac (or the large angle into the initial linac could be reduced). A much longer linac design would not fit easily within this relatively confined space. The degree of curvature that could be added is limited by the fact that $\mathrm{H}^{-}$ions must be accelerated and transported to the MI, and the bending fields must be low enough to avoid magnetic stripping.

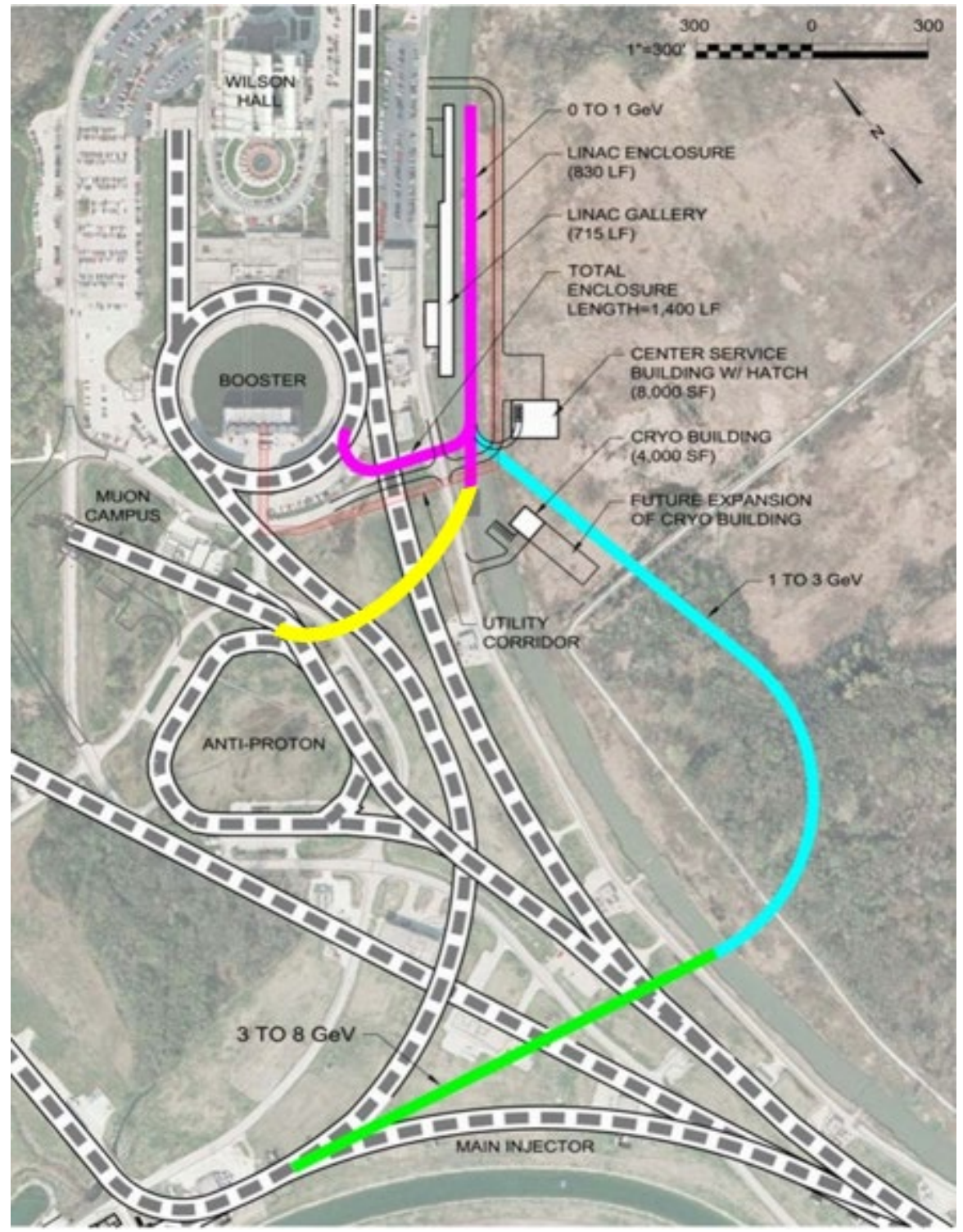

FIGURE 1. Layout on the $8 \mathrm{GeV}$ Linac as envisioned in Project X (from ref. 3).

In the Project $\mathrm{X}$ design, the $3 \mathrm{GeV}$ linac was designed to feed high-intensity Kaon physics experiments. In earlier versions the $\mathrm{cw}$ linac went only to $2 \mathrm{GeV}$, which was adequate for some experiments, but was inefficient in Kaon production. A high-intensity Kaon program may not be as important as in 2012, so this transition point could be reevaluated. The MI is intrinsically pulsed and needs the Linac for only 26 ma-ms per $1.2 \mathrm{~s}$. It is expected that 1300 $\mathrm{MHz}$ mass-produced pulsed ILC cryomodules would be much cheaper than alternatives, which would need additional development. Therefore the $3 \rightarrow 8 \mathrm{GeV}$ Linac was initiated as a pulsed Linac design.

The $8 \mathrm{GeV}$ beam will also have some other functions. It could feed a continuation of the present short-baseline neutrino experiments, which currently use $8 \mathrm{GeV}$ Booster beam. It could also be a primary beam source for a 
continuation of the g- 2 experiment or other experiments. The pulsed linac could provide $\sim 400 \mathrm{~kW}$ to such experiments. The Fermilab Project $\mathrm{X}$ also considered conversion of this linac to cw mode as a future upgrade, which could then provide up to $\sim 8 \mathrm{MW}$ for ultra high-intensity applications such as a "neutrino factory".

The MI ring is partnered with a same circumference Recycler ring (RR). The recycler ring consists of permanent magnets, fixed to $8 \mathrm{GeV}$ proton energy. In the present MI operation, protons are collected in the RR during the MI acceleration cycle, to be injected into the MI at the beginning of its accelerator cycle. The same mode of operation could be adopted in PIP-III, for both linac and RCS scenarios.

The aperture and acceptance of the RR is a bit smaller than the MI ( $24 \pi$ versus $\sim 30 \pi, 95 \%$, normalized), so use of it restricts MI intensity. Also the injection is fixed to $8 \mathrm{GeV}$. A higher energy injection would increase that acceptance, following a factor of $\beta \gamma$.

In the Linac scenario, beam could be injected directly into the MI in a single 26 ma-ms injection pulse (13ms at $2 \mathrm{ma}$ ); but, as discussed below, stripper foil heating is increased. For an RCS, multiple RCS pulses are required to feed the MI, which would then require an extended injection time, which would reduce the total intensity delivered by the MI. (Accumulation in the RR from the RCS avoids that extension.)

\begin{tabular}{|l|l|l|l|l|l|}
\hline \multicolumn{1}{|c|}{ Section } & $\begin{array}{l}\text { Freq, } \\
\text { MHz }\end{array}$ & $\begin{array}{l}\text { Energy } \\
\text { MeV }\end{array}$ & Cav/mag/CM & $\begin{array}{l}\text { CM length, } \\
\mathbf{m}\end{array}$ & \multicolumn{1}{|c|}{ Type } \\
\hline HWR $\left(\beta_{\mathrm{G}}=0.11\right)$ & 162.5 & $2.1-10$ & $8 / 8 / 1$ & $6.2 \mathrm{~m}$ & SSR, solenoid \\
\hline SSR1 $\left(\beta_{\mathrm{G}}=0.22\right)$ & 325 & $10-32$ & $16 / 10 / 2$ & 5.2 & SSR, solenoid \\
\hline SSR2 $\left(\beta_{\mathrm{G}}=0.47\right)$ & 325 & $32-160$ & $35 / 20 / 7$ & 6.5 & SSR, solenoid \\
\hline LB 650 $\left(\beta_{\mathrm{G}}=0.61\right)$ & 650 & $160-520$ & $36 / 18 / 9$ & 5.2 & 5-cell elliptical, doublet \\
\hline HB 650 $\left(\beta_{\mathrm{G}}=0.9\right)$ & 650 & $520-820$ & $24 / 8 / 4$ & 9.5 & 5-cell elliptical, doublet \\
\hline HB 650 $\left(\beta_{\mathrm{G}}=0.9\right)$ & 650 & $820-3000$ & $132 / 22 / 22$ & 9.5 & 5-cell elliptical, quad \\
\hline ILC 1.3 $\left(\beta_{\mathrm{G}}=1.0\right)$ & 1300 & $3000-8000$ & $224 / 28 / 28$ & 12 & 9-cell elliptical, quad \\
\hline
\end{tabular}

Table 1: Parameters of the Project X 8 GeV Linac

\section{Alternate scenarios}

The baseline scenario of $3 \mathrm{GeV} \mathrm{cw}$ linac and pulsed $3 \rightarrow 8 \mathrm{GeV}$ linac is still somewhat of a mismatch. The physics need for a multi-MW $3 \mathrm{GeV}$ cw linac is unclear and, on the other hand, a pulsed linac could limit further expansion. Alternative scenarios are possible.

One possibility would be extending the $650 \mathrm{MHz} \mathrm{cw}$ Linac up to $8 \mathrm{GeV}$, simply extending the $1 \rightarrow 3 \mathrm{GeV}$ lattice and cryomodules up to $8 \mathrm{GeV}$. The initial 6-cell, $9.5 \mathrm{~m}$ cryomodules are designed to accelerate at better than 100 $\mathrm{MeV}$ per cryomodule; at the $100 \mathrm{MeV}$ spec this requires 50 cryomodules for a $\sim 500 \mathrm{~m}$ linac, somewhat longer than the $390 \mathrm{~m}$ initially allotted. This would be difficult to accommodate in the space constraints. $650 \mathrm{MHz}$ cavity prototypes are performing significantly better than specification, with improved cavity processing including nitrogen doping; accelerating gradients of $25 \mathrm{MV} / \mathrm{m}$ are readily obtained, much more than the $17.6 \mathrm{MV} / \mathrm{m}$ spec. One should thwn be able to obtain $\sim 125 \mathrm{MeV} /$ cryo, which would reduce the linac to 40 cryos or $\sim 400 \mathrm{~m}$.

Another possibility is shifting the cw to pulsed transition down toward the PIP-II energy. At Project X specs, a $1 \rightarrow 8 \mathrm{GeV}$ pulsed linac would require $\sim 401300 \mathrm{MHz}$ cryos $(\sim 495 \mathrm{~m})$, which is much shorter than the complete project X linac length. Mass production of ILC $1300 \mathrm{MHz}$ cavities could make this an affordable option; perhaps competitive with RCS options. (Project X estimates had the $1300 \mathrm{MHz}$ at $\sim 1 / 2$ the cost of $650 \mathrm{MHz}$ systems.)

\section{Magnetic stripping constraints}

The $8 \mathrm{GeV}$ Linac beam must be transmitted as $\mathrm{H}^{-}$, for compatibility with $\mathrm{H}^{-}$injection into the Recycler, and the bending fields in the $8 \mathrm{GeV}$ PIP-III transports are limited to $\sim 0.05 \mathrm{~T}$ to avoid magnetic stripping to $\mathrm{H}^{0}$. The stripping time can be estimated using the formula of Schrek: 


$$
\tau=\frac{a}{3.197 B_{t} p} \exp \left(\frac{b}{3.197 B_{t} p}\right) \text { seconds, }
$$

where $p=8.89 \mathrm{GeV} / \mathrm{c}$ for $8 \mathrm{MeV} \mathrm{H}^{-}$, B and $a$ and $b$ are parameters fitted from data. [4, 5] Keating et al. obtained $a=$ $3.07310^{-14}$ and $b=44.14$ from $800 \mathrm{MeV}$ data. [6] At these parameters $\tau=0.64 \mathrm{~s}$ or $\left(\beta \gamma \mathrm{c} \tau=1.8 \times 10^{9} \mathrm{~m}\right)$. Earlier Project X versions, which were located deep within the Main Ring circle, had a very long $8 \mathrm{GeV}$ transport (several hundred meters) In our current geometry, up to $\sim 0.07 \mathrm{~T}$ could be allowed, with losses of $\mathrm{H}^{-}$remaining $<\sim 10^{-5}$.

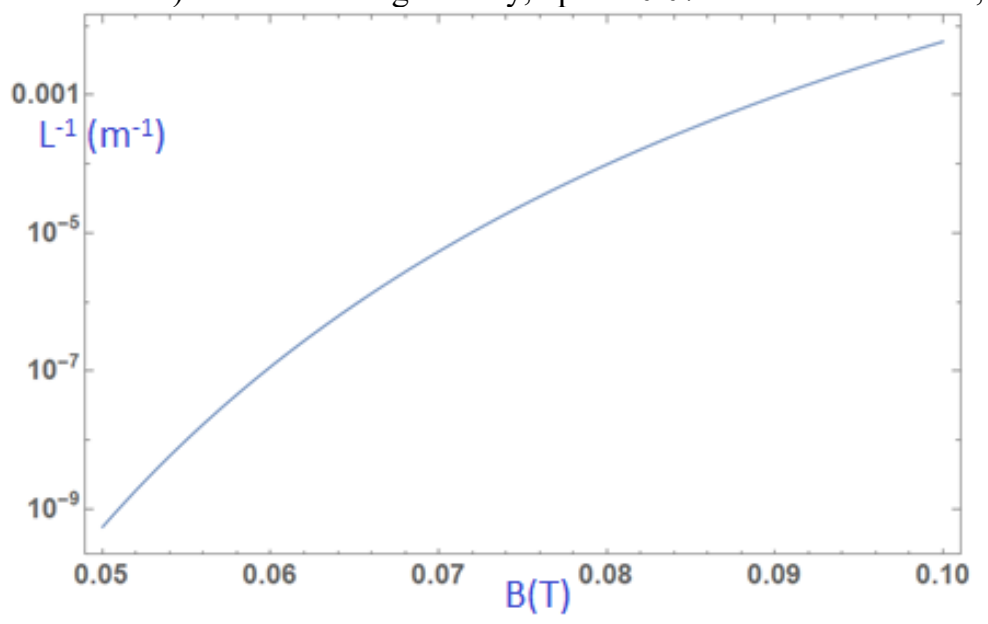

Figure 2: Magnetic stripping rate $\left(\mathrm{m}^{-1}\right)$ as a function of $\mathrm{B}(\mathrm{T})$ for $8 \mathrm{GeV} \mathrm{H}^{-}$.

In the geometry of fig. 1, a much longer bend occurs at the end of the cw linac, which was at $3 \mathrm{GeV}$. At this momentum, $\mathrm{B}<0.15 \mathrm{~T}$ is required, at which the stripping rate is $4 \times 10^{-10} \mathrm{~m}^{-1}$, so that the stripping losses are less than $10^{-7}$.

\section{Stripping Foil efficiency}

In the foil the $\mathrm{H}^{-}$ions are stripped to $\mathrm{H}^{0}$ and $\mathrm{H}^{+}$, and $\mathrm{H}^{0}$ ions are stripped to $\mathrm{H}^{+}$. Equations for stripping versus foil thickness have been developed by Gulley et al.,[7] from fits to measured stripping data. The equations are:[8]

$$
\begin{aligned}
& f_{H^{-}}(t, \beta)=\operatorname{Exp}\left[-(0.479+0.0085) \cdot 0.05 t / \beta^{2}\right] \\
& f_{H^{0}}(t, \beta)=\frac{0.479}{(0.479+0.0085-0.187)}\left(\operatorname{Exp}\left[-(0.187) \cdot 0.05 t / \beta^{2}\right]-\operatorname{Exp}\left[-(0.479) \cdot 0.05 t / \beta^{2}\right]\right) \\
& f_{H^{+}}(t, \beta)=1-f_{H^{0}}(t, \beta)-f_{H^{-}}(t, \beta)
\end{aligned}
$$

where $\beta=v / c$ is the usual kinematic factor for the incident $\mathrm{H}^{-}, t$ is the carbon foil thickness in $\mu \mathrm{gm} / \mathrm{cm}^{2}$. For a $500 \mu \mathrm{gm} / \mathrm{cm}^{2}$ thick foil, $98.6 \%$ of initial $\mathrm{H}^{-}$are stripped to $\mathrm{H}^{+}$(protons). For graphite (at $\rho=2.0 \mathrm{gm} / \mathrm{cm}^{3}$ ), this is a $2.5 \mu \mathrm{m}$ thick foil, or $1.4 \mu \mathrm{m}$ thick for diamond $(\rho=3.6)$. Figure 2 shows the variation of ion fraction through a foil with thickness of $500 \mu \mathrm{g} / \mathrm{cm}^{2}$. 


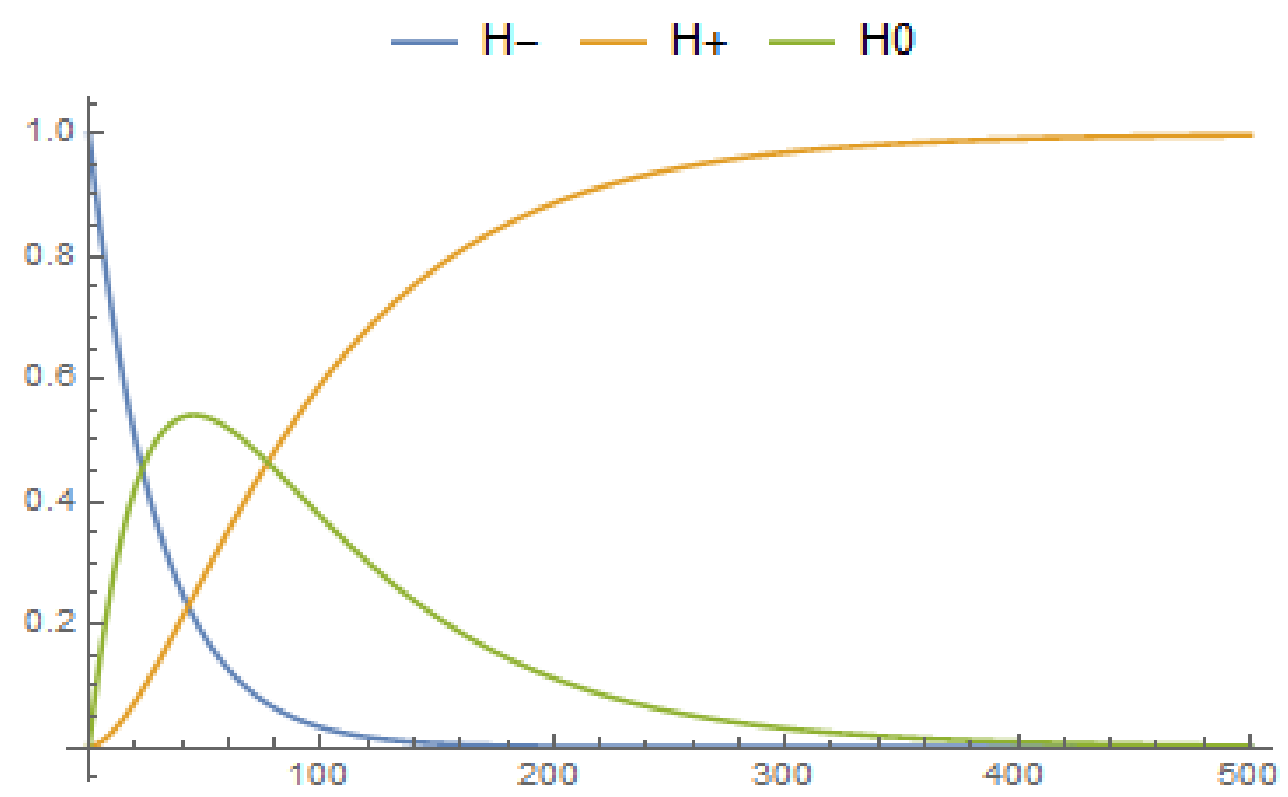

FIGURE 3. Fraction of beam that is $\mathrm{H}^{-}, \mathrm{H}^{0}$, or $\mathrm{H}^{+}$as it passes through a $\mathrm{C}$ foil with final thickness of $500 \mu \mathrm{g} / \mathrm{cm}^{2}$. At 400 $\mu \mathrm{g} / \mathrm{cm}^{2}$, the beam is $\sim 96.4 \% \mathrm{H}^{+}$, and $3.6 \% \mathrm{H}^{0}$. At $500 \mu \mathrm{g} / \mathrm{cm}^{2}$, it is $\sim 98.6 \% \mathrm{H}^{+}$. At $600 \mu \mathrm{g} / \mathrm{cm}^{2}$, it would be $\sim 99.5 \% \mathrm{H}^{+}$.

It is important that the multiple scattering caused by the foil be small compared to the emittance of the beam. The normalized emittance of the PIP-II $\mathrm{H}^{-}$injected beam is $\sim 0.3 \mathrm{~mm}-\mathrm{mrad}$. The multiple scattering increase in the emittance is given by: $\quad \Delta \varepsilon_{N} \cong \frac{\beta_{T}(13.6)^{2} t}{2 \beta^{2} P_{\text {beam }} m_{\text {beam }} X_{0}}$,

where $P_{\text {beam }}=8889 \mathrm{MeV} / \mathrm{c}, m_{\text {beam }}=938 \mathrm{MeV} / \mathrm{c}$, and $X_{0}=42.7 \mathrm{gm} / \mathrm{cm}^{2}$, the radiation length for carbon (C). For $t$ $=0.0005 \mathrm{gm} / \mathrm{cm}^{2}$ and a focusing betatron function of $\beta_{T}$ of 70 , we obtain $\Delta \varepsilon_{N}=\sim 0.009 \mathrm{~mm}-\mathrm{mrad}$ in a single turn. This effect is magnified by the number of turns of particle passage through the foil, which is $\sim 100$ in a typical scenario, and that increases $\Delta \varepsilon_{N}$ to $\sim 1 \mathrm{~mm}$-mrad. This is larger than the injected emittance but smaller than the accumulated inject emittance ( $\sim \mathrm{mm}$-mrad ). The effect is significant but probably tolerable. The scattering may also cause some losses that must be considered in controlling activation in the ring or near the injection area.

Energy loss for protons in graphite is $\sim 4.0 \mathrm{MeV} / \mathrm{cm}$ or $\sim 1 \mathrm{keV}$ in a $2.5 \mu \mathrm{m}$ foil; this is $\sim 10^{-7}$. Increase in energy spread is an order of magnitude smaller. The beam energy spread is relatively unaffected by passage through the foil.

\section{Beam Transport and stripper location}

The baseline scenario for injection into the MI or Recycler is the MI10 area, which will have to be modified to accommodate stripper foil injection. Betatron functions in that region are displayed in figure 4.[9] The betatron functions are somewhat enlarged to $70 \mathrm{~m}$ by $30 \mathrm{~m}$ from that in the rest of the recycler ring. This reduces the foil heating by reducing the density of the hits on foil. Figure 5 shows the injection kicker geometry used to paint the injected beam through the foil. 


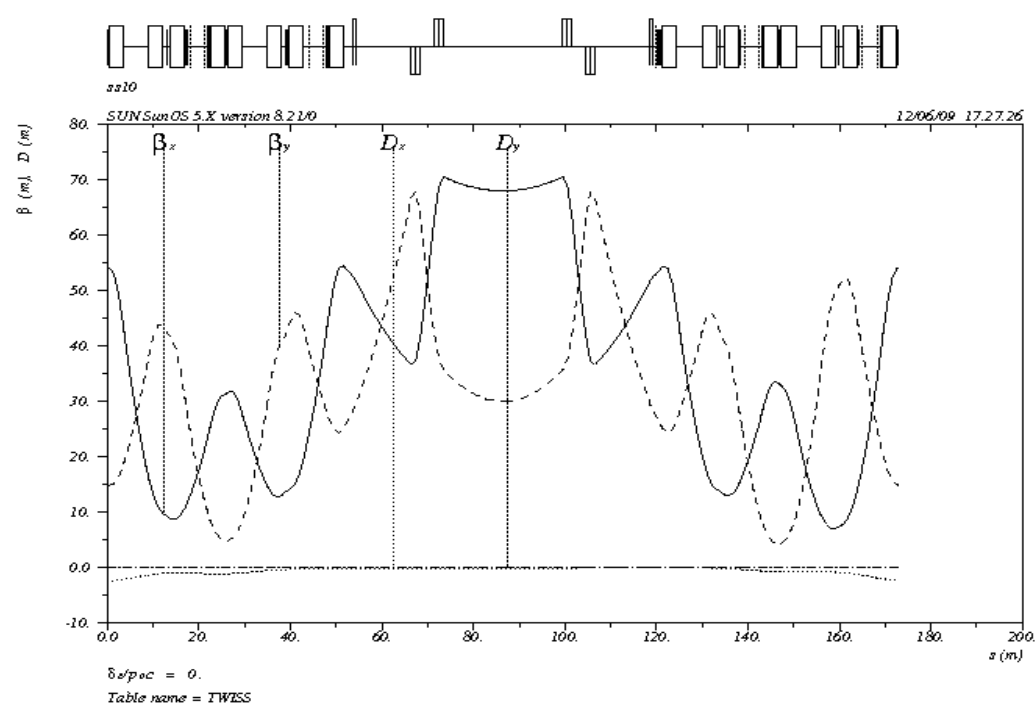

Figure 4. Betatron functions in the Recycler injection region (from ref. 2)

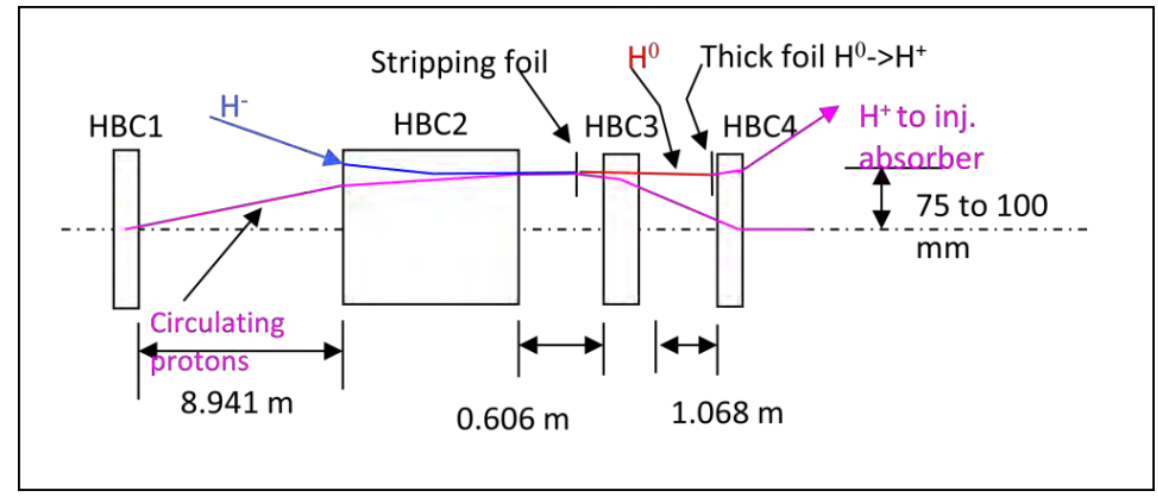

Figure 5: Injection insert components to be placed in the large $\beta$ straight section shown above.

\section{FOIL HEATING ESTIMATES}

The beam stripping would heat the foil, and foil heating has been a significant problem in foils for multi-turn stripping injection into synchrotrons. The large number of injection turns implies multiple particle hits on the foils which greatly increases the heating effects, and modeling of multiturn injection is relatively uncertain. SNS and JParc have had the most experience with multiturn injection and have foil heating problems. These problems could be significantly more for the PIP-III to RR line since the injected beam is relatively high intensity and is initially concentrated onto a very small spot on the foil.

SNS and J-Parc have had the most experience with multiturn injection and have foil heating problems. Fig. 6 shows a successful SNS production foil after 4 months of use, at up to 1MW beam power. The foil shows large distortion and damage; many foils fail completely. The best performance has been from nanocrystalline diamond foils produced in Oak Ridge. While foil temperatures are not directly measured in operation, foil lifetimes are compromised when peak operating temperatures are calculated to be greater than $\sim 1600^{\circ} \mathrm{K}$. 

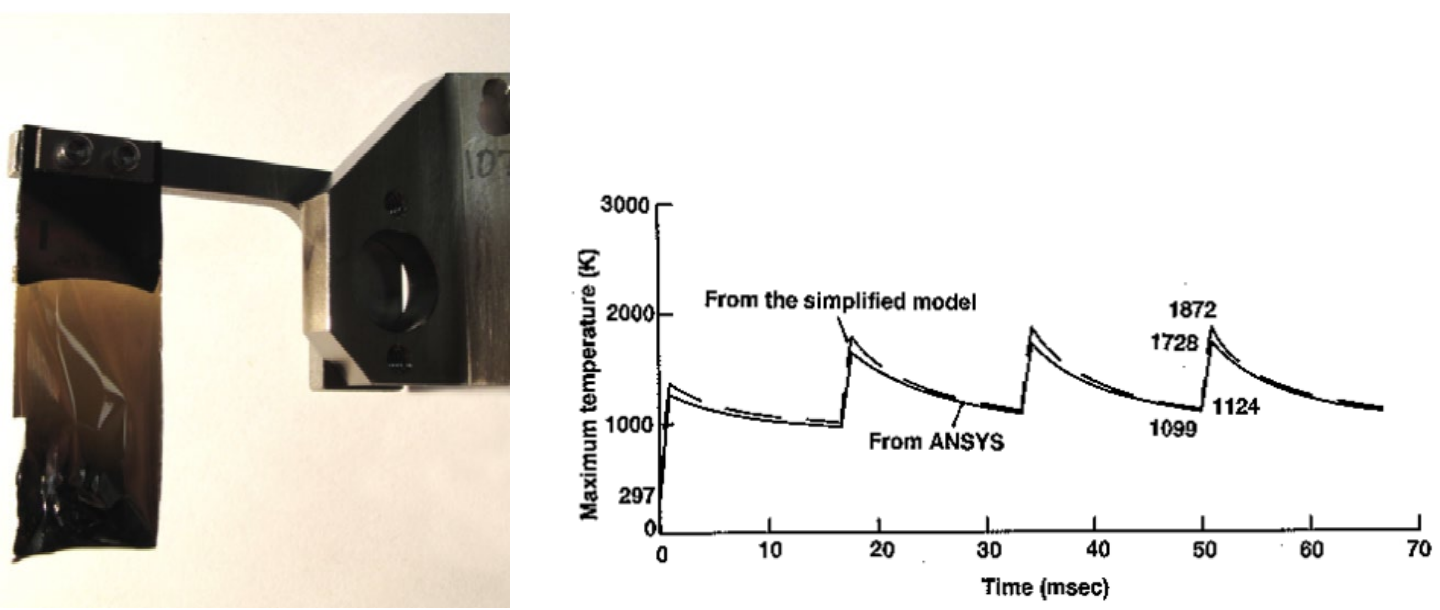

Figure 6 Stripper foil after use in SNS (left) . Calculated Temperature of SNS stripper foil as a function of time. The stripper foil temperature at SNS spikes to $\sim 1800{ }^{\circ} \mathrm{K}$ at the $60 \mathrm{~Hz}$ pulse frequency, with an equilibrium of $\sim 1100$ ${ }^{\circ} \mathrm{K} .[10]$ (right)

Foil sublimation occurs at high temperatures and increases greatly at higher temperatures. An equation for that sublimation is:

$$
\frac{1}{\rho} \frac{d h(T)}{d t}=-8.12 \times 10^{8} \frac{e^{-\frac{83500}{T}}}{\sqrt{T}}
$$

where the result is in $\mathrm{m} / \mathrm{s}, \mathrm{T}$ is temperature in ${ }^{\circ} \mathrm{K}$, and $\rho$ is in $\mathrm{gm} / \mathrm{cm}^{3}$. The factor is plotted in fig. The effect must be integrated over the injection period. This effect becomes significant for $\mathrm{T}$ greater than $\sim 1800{ }^{\circ} \mathrm{K}$, and would preclude use at $\mathrm{T}>\sim 2000^{\circ} \mathrm{K}$.

The temperature heating can be estimated using equations presented by Liaw et al.[10] and Drozhdin et al.[11] In the approximation of only radiative cooling, the evolution of temperature at the center of the incident beam (defined by the central density $\left.1 /\left(4 \pi \sigma_{x} \sigma_{y}\right)=1 / A\right)$.

$$
\rho t_{f} C_{h} \frac{d T}{d t} \cong \frac{d E_{H^{-} \rightarrow p}}{d s} \frac{t_{f}}{A} \frac{n_{h} I}{e}-2 \varepsilon \sigma_{B}\left(T^{4}-T_{0}^{4}\right)
$$

where $T(t)$ is the temperature, $\rho$ is the density, $C_{h}$ is the heat capacity, which is a function of temperature for graphite, $t_{f}$ is the foil thickness, $d E / d s$ is the energy loss (which includes contributions from the proton and electrons), $I / e$ is the injected beam current, $n_{h}$ refers to the average number of turns the average proton passes through the foil, A is the area of the beam spot on foil, $\varepsilon$ is the efficiency ( 0.8 for graphite), $\sigma_{B}$ is Boltzmann's constant, $T_{0}$ is the background temperature $\left(297^{\circ} \mathrm{K}\right)$. Some typical numbers associated with PIP-II are $I=0.002 \mathrm{~A}$, $t=0.000003 \mu\left(600 \mu \mathrm{g} / \mathrm{cm}^{2}\right), d E / d s=\sim 4 \mathrm{MeV} / \mathrm{cm}, \rho=2 \mathrm{gm} / \mathrm{cm}^{3}$ (C target), $C_{h} \cong 12+2.87 T-0.00145 T^{2}+3.110^{-7} T^{3}$ $-2.410^{-11} T^{4} \mathrm{~J} / \mathrm{kg} /{ }^{\circ} \mathrm{K}, \sigma_{B}=5.6710^{-8} \mathrm{~W} / \mathrm{m}^{2} \mathrm{~K}^{4}$. (The factor $\mathrm{n}_{\mathrm{h}} \mathrm{I} / \mathrm{A}$ can be recharacterized as the maximum hit density on the foil, and can be determined in a simulation of the painting scenario.)

The parameter that is can vary greatly in injection scenarios is the maximum hit density, which depends on injection geometry and optics and foil/beam painting strategy. The strategy used is to minimize $\mathrm{n}_{\mathrm{h}}$ while matching the accumulated beam to fill the aperture as fully and evenly as possible. The injected beam size is a small fraction of the accumulated beam size. Raparia[12] estimated an optimum for $\mathrm{n}_{\mathrm{h}}$ where $\mathrm{n}_{\mathrm{h}}=\mathrm{n}_{\text {turns }} \times\left(\varepsilon_{\text {injected }} / \varepsilon_{\text {acceptance }} / 4\right)$, where $\mathrm{n}_{\text {turns }}$ is the total number of injected turns, and the area $\mathrm{A}$ used would be $\sim 6 \pi \sigma_{\mathrm{x}} \sigma_{\mathrm{y}}$ of the injected beam, which assumes the foil location is matched to the injected beam size. A more accurate result can be obtained by tracking the beam through the injection process and calculating the maximum hit density, to obtain the hottest spot on the foil.

The simplified model of eq. (2) does not include conduction and other effects; also the temperature distribution across the foil is not calculated. 
Table 2 : Injection cases and parameters; baseline injected current of 2 ma for PIP-III

\begin{tabular}{|l|l|l|l|l|l|}
\hline & SNS & PIP-II & PIP III RCS-A & PIP III RCS-B & PIP III Linac A \\
\hline Foil thickness & $350 \mu \mathrm{g} / \mathrm{cm}^{2}$ & 400 & 600 & 600 & 600 \\
\hline Injection energy & 1.0 & 0.8 & 1.0 & 2.0 & $8.0 \mathrm{GeV}$ \\
\hline $\begin{array}{l}\text { Ring } \\
\text { circumference }\end{array}$ & 248 & 474 & 600 & $553 \mathrm{~m}$ & $3319.4 \mathrm{~m}$ \\
\hline Injection time & $1 \mathrm{~ms}$ & $0.55 \mathrm{~ms}$ & $2.17 \mathrm{~ms}$ & 2.1 & 12.5 or $6 \times 2.1 \mathrm{~ms}$ \\
\hline Injection current & $18.6 \mathrm{ma}$ & $2 \mathrm{ma}$ & 2 & 2 & 2 \\
\hline Injection turns & 1060 & 293 & 950 & 1080 & 1125 or $6 \times 189$ \\
\hline Intensity $(\mathrm{Np})$ & $1.16 * 10^{14}$ & $6.6 \times 10^{12}$ & $2.6 \times 10^{13}$ & $2.6 \times 10^{13}$ & $1.6 \times 10^{14}$ \\
\hline$\beta, \beta \gamma$ & $0.875,1.80$ & $0.842,1.56$ & $0.875,1.80$ & $0.945,2.97$ & $0.995,9.47$ \\
\hline$\beta_{\mathrm{x}}, \beta_{\mathrm{y}}$ & $17.4,4.56$ & $6.3,20.0$ & 12,30 & $20.5,20.5$ & $30,70 \mathrm{~m}$ \\
\hline$\varepsilon_{\mathrm{n}, 95 \%,}\left(6 \varepsilon_{\mathrm{n}, \sigma}\right)$ & 6 & 16 & 24 & 22 & 24 \\
\hline Peak Hits $/ \mathrm{mm}^{2}$ & & $4.16 \times 10^{12}$ & $2.5 \times 10^{13}$ & $5.7 \times 10^{13}$ & $2.63 \times 10^{14}$ \\
\hline
\end{tabular}

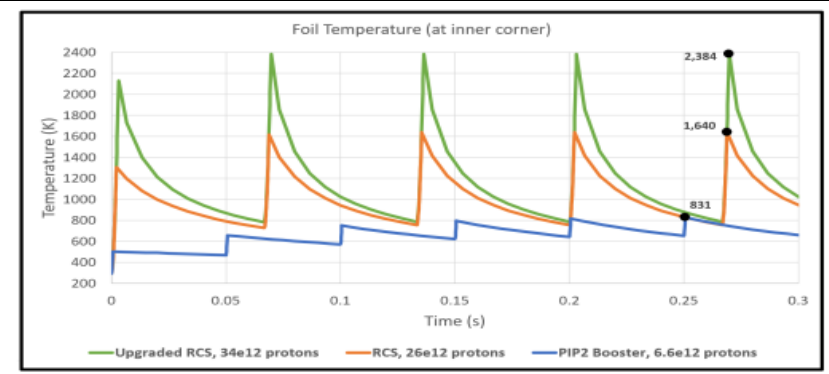

Figure 7. Results of calculation of foil heating for various RCS scenarios. PIP-II and 26e12 RCS injection are within tolerable limits. The high intensity $34 \mathrm{e} 12$ case is not, and would require a reoptimization of the injection parameters and/or innovations such as laser assisted stripping ( From ref. 13.)

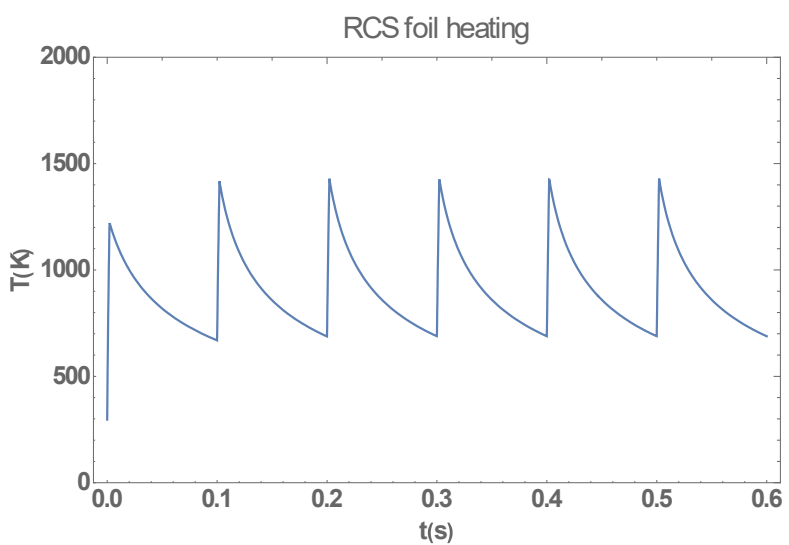

Figure 8. Calculation for foil heating for the PIP-II+ RCS of ref. 14.

We have also considered some evaluations of foil heating for RCS injections. These cases include the SNS linac/accumulator, PIP-II injection into the Booster, and two versions of an RCS for injection into the RR/MI [13, 14]. We have done this to improve our understanding of the injection process and to benchmark our calculations against other similar calculations. The RCS cases will have much higher temperatures than both the previously established current Booster injection and the future PIP-II injection. The expected temperatures are similar to those experienced at SNS.

Drozhdin et al. have analyzed the topic of $8 \mathrm{GeV}$ linac-based RR injection in significant detail and we will summarize and discuss their results. They presented a detailed painting scenario and tracked particle motion in the injection geometry to determine more precisely a maximum density of hits on targets. With that calculation the heating calculation can be rewritten as: 


$$
\rho t_{f} C_{h} \frac{d T}{d t} \cong \frac{d E_{p} t_{f}\left(1-\delta_{e}\right)}{d s} N^{\prime}(t)-2 \varepsilon \sigma_{B}\left(T^{4}-T_{0}^{4}\right)
$$

where $\mathrm{N}^{\prime}(\mathrm{t})$ is the maximum hit density rate on the foil. From simulation, they obtained an integrated hit density of $5.15 \times 10^{14} / \mathrm{mm}^{2}$ for a $1 \mathrm{ma}-25.7 \mathrm{~ms}$ injection.

The factor $\delta_{\mathrm{e}}$ results from their evaluation of the energy deposition in the foil. Much of the energy lost by protons in the foil is in the form of higher energy electrons, which escape the thin foil and do not heat it. This correction factor is $\sim 0.28$ for a $600 \mu \mathrm{g} / \mathrm{cm}^{2}$ foil, with $8 \mathrm{GeV}$ protons. (The correction factor is not included in some analyses.)

Fig. 9 shows some results of their analyses, which we have been able to duplicate in Mathematica simulations. In the $1 \mathrm{ma}-25.7 \mathrm{~ms}$ injection the foil is heated to $2350 \mathrm{~K}$ in each injection, which is probably unacceptably high. They developed other scenarios, based on splitting the injection into 6 separate injections, spaced $0.1 \mathrm{~s}$ apart. This allows the foil to cool between injections, and therefore reduces the peak temperature. With a 1 ma injector the peak $\mathrm{T}$ is still over $2000 \mathrm{~K}$, which is undesirable. However a 2 ma injector would reduce the peak $\mathrm{T}$ to $\sim 1500 \mathrm{~K}$, which is relatively manageable, and within parameters previously demonstrated. A 4 ma injection would reduce that to $\sim 1000 \mathrm{~K}$, providing further safety margin and potential future intensity increase.

The $2 \mathrm{ma}-2.16 \mathrm{~ms} \times 6$ scenario could be chosen as a baseline injection scenario to be considered in an initial Linac based PIP-II+ reference design.

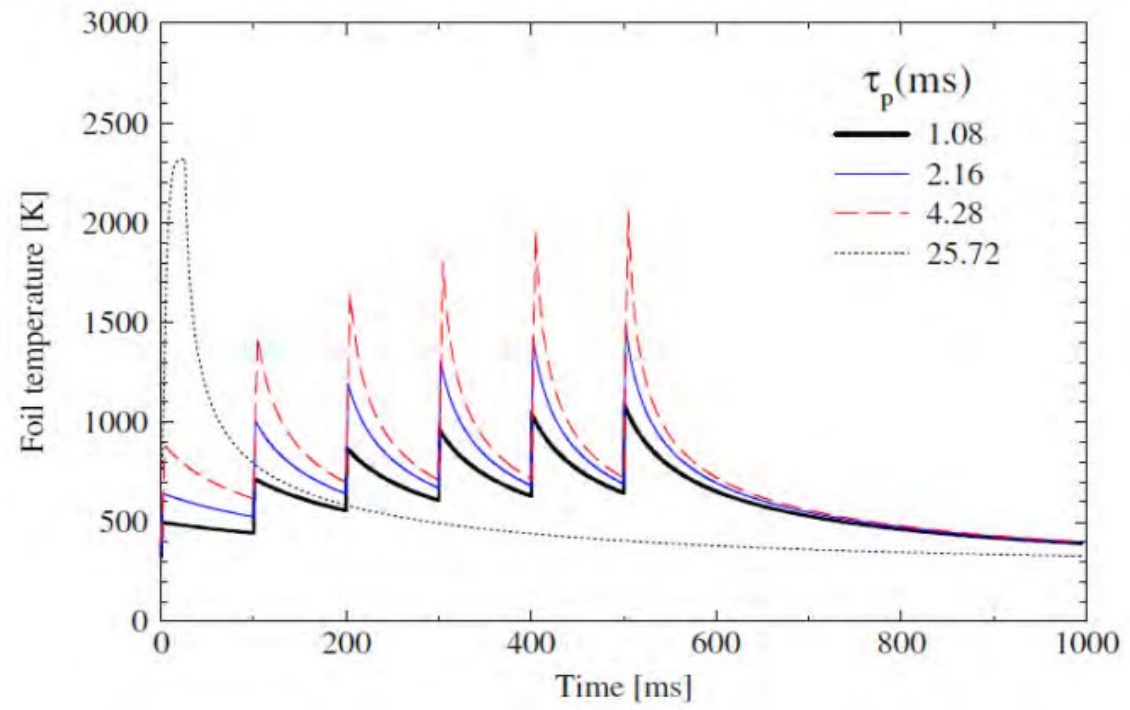

Figure 9. Temperatures of the foil for injection from an $8 \mathrm{GeV}$ Linac into the Recycler using $600 \mu \mathrm{gm} / \mathrm{cm}^{2}$ carbon foil for various painting cycle variations. Total protons accumulated is the same, peak current was changed from 1 to 2 to 4 ma. (from ref. 11) 


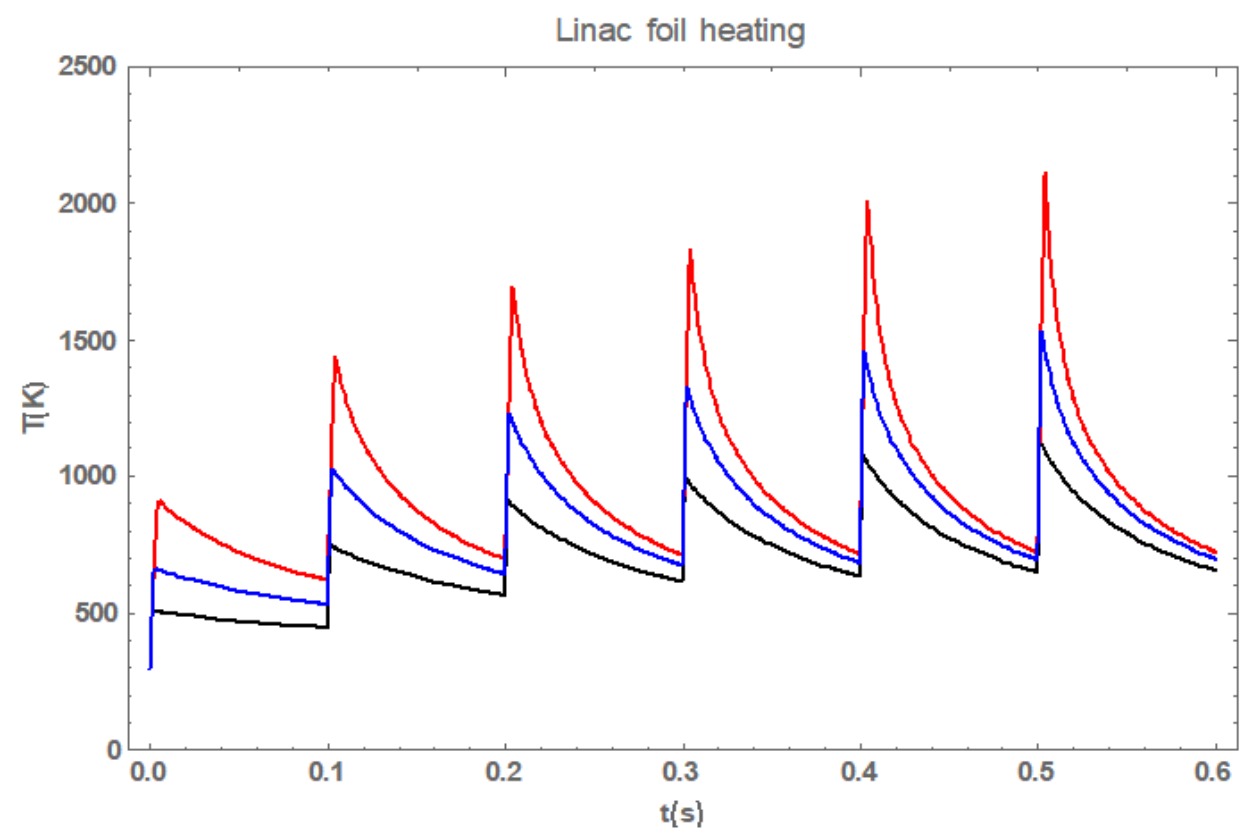

Figure 10: Same parameters as figure 9, but calculated in a Mathematica model. Close agreement indicates that we have correctly interpreted the parameters and methods of ref. [11], and can modify the model to consider variations in injection scenarios.

\section{Variations of the Injection scenario}

Using our Mathematica model, we considered other scenarios. With the injection current increased to 2 or 4 ma, a single injection with $\sim 13 \mathrm{~ms}$ or $6.5 \mathrm{~ms}$ length would obtain the same intensity, with $1 / 2$ or $1 / 4$ the number of hits on foil. The peak temperature is reduced to $\sim 2300 \mathrm{~K}$ with $2 \mathrm{ma}$ and to $\sim 1850 \mathrm{~K}$ with $4 \mathrm{ma}$. The $4 \mathrm{ma}$ case is close to acceptable. A single injection of that scale would enable direct injection into the MI, without increasing the MI cycle time. The larger aperture of the MI would enable injection into a larger emittance beam, which would reduce the peak temperature a bit more and may enable higher intensity. Much higher intensity would be limited by the foil heating, however.

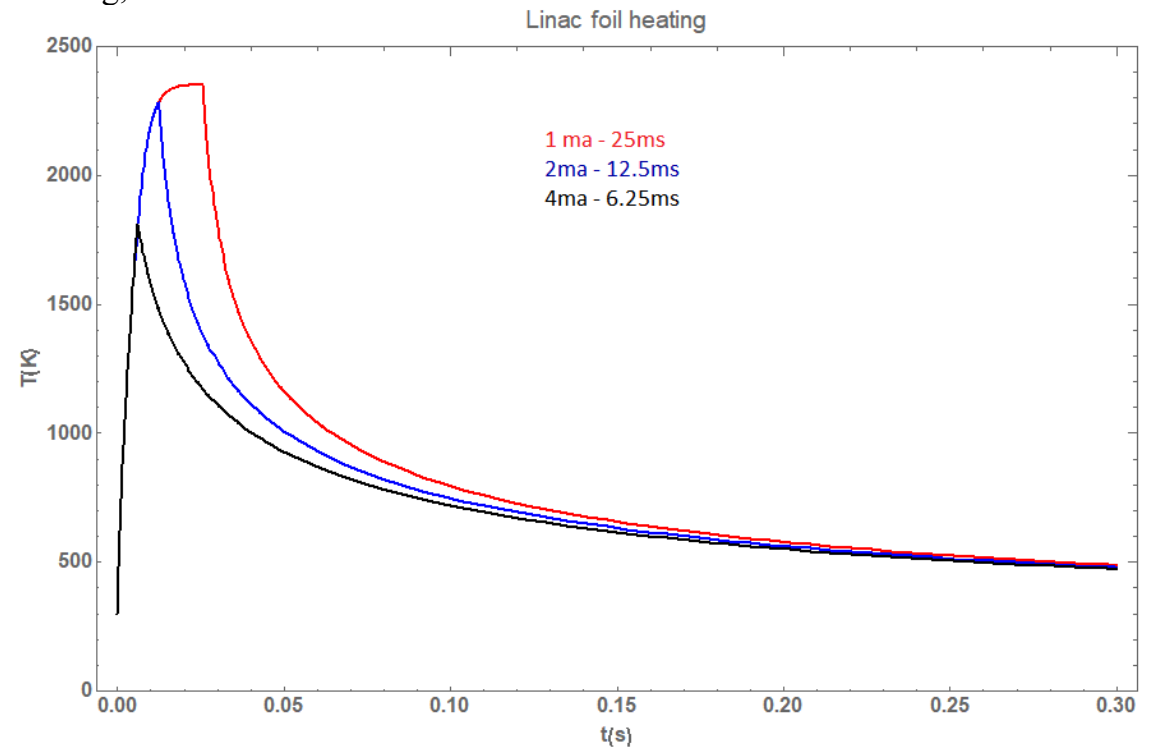

Figure 11: Three injections scenarios using a single linac pulse. Peak current was increased from 1 to 2 to 4 ma, shortening the injection cycle length, and therefore the number of turns. The last case (4 ma) approaches acceptability restrictions. 
While PIP-II is designed to provide $2 \mathrm{ma} \mathrm{cw}, 4 \mathrm{ma}$ or higher (pulsed) beam for PIP-III may require modification of the PIP-II linac systems. RCS scenarios are also considering increasing the pulsed current to similar levels, for similar reasons; they note that this will require rf power modifications.

\section{Future Variations and Studies}

Some other variations on these scenarios could be developed. Reduction of the foil thickness to $400-500$ $\mu \mathrm{gm} / \mathrm{cm}^{2}$ reduces heating proportionally. Placing the foil at an angle could improve the effective emissivity of the foil. Larger beam size in the foil region could be enabled with a larger $\beta_{\mathrm{x}}, \beta_{\mathrm{y}}$.

In practice, the hot spot should be moved around the foil (or the foil moved) to minimize localized foil distortion. A particularly desirable configuration could be a rotating foil, such as that used by Hasabe et al. for U stripping[15] at RIKEN and was also studied for the Proton Driver by Johnson and Tang.[16] These variations may be needed if a higher power PIP-III beam (for a $5 \mathrm{MW}$-scale MI) were using stripping foils.

Another option is to use laser assisted stripping.[17, 18] This is under development at SNS and could also be used as an upgrade for RCS systems. Laser stripping may be much easier with $8 \mathrm{GeV}$ proton beam, since the incident light is Doppler shifted into higher energies in the beam frame. Infrared lasers, which are much more readily available, can be used instead of the UV light required for $1-2 \mathrm{GeV}$ stripping.

\section{ACKNOWLEDGMENTS}

This manuscript has been authored by Fermi Research Alliance, LLC under Contract No. DE-AC02-07CH11359 with the U.S. Department of Energy, Office of Science, Office of High Energy Physics.

\section{REFERENCES}

1. M. Ball et al., The PIP-II Conceptual Design Report (2017).

2. Project X Accelerator Reference Design, Physics Opportunities, Broader Impacts, June 2013 Fermilab TM-2557.

3. S. Holmes, Proton Improvement Plan II, presentation to Fermilab Users meeting, June 11, 2014

4. W. Chou et al., "8 GeV H- ions: transport and injection," Proc. PAC 2005, Knoxville, TE, p. 1222 (2005).

5. L.R. Scherk, Canadian J. of Phys, 57, 558 (1979).

6. P.B. Keating et al., Phys. Rev. A 52, 4547 (1995).

7. M. S. Gulley et al., Physical Review A 53, 3201-3210 (1996).

8. M. Plum, "Stripper Foils for H- Beams", in Handbook of Accelerator Physics and Engineering, second edition, A. W. Chou, K. H. Weiss, Tigner and F. Zimmermann, eds., p. 574 (2013).

9. D. E. Johnson, "Project X: $8 \mathrm{GeV}$ Recycler Injection Project X doc. 74 (2008)

10. C. J. Liaw et al., Proc PAC 1999, New York, NY, p. 3300 (1999).

11. A. I. Drozhdin, I. L. Rakhno, S. I. Striganov, and L. G. Vorobiev, Phys. Rev. STAB 15, 011002 (2012).

12. D. Raparia, "Foil Issues", Project X-doc-701 Sept. 2010.

13. J. Eldred, V. Lebedev, and A. Valishev, Rapid-Cycling Synchrotron for Multi-Megawatt Proton Facility at Fermilab", arXiv:1903.12408v2, JINST 14 P07021 (2019).

14. S. Nagaitsev and V. Lebedev, "A Cost-effective Rapid-cycling Synchrotron”, Fermilab-Pub-18-546-AD-DI,

15. H. Hasebe et al., "Development of a rotating graphite carbon disk stripper" AIP Conf. Proc. 1962, 030004 (2018).

16. D. Johnson and Z. Tang, "Rotating Foil and Stripping Station Studies", unpublished (2012).

17. I. Yamane, H- charge-exchange injection without hazardous stripping foils, Phys. Rev. Accel. Beams 1, 053501 (1998).

18. S. Cousineau et al, "High efficiency laser-assisted $\mathrm{H}^{-}$charge exchange for microsecond duration." Phys. Rev. Accel. Beams 20, 120402 (2017). 